\section{(A) Check for updates}

Cite this: Analyst, 2021, 146, 3273

\title{
Detection of low glucose levels in sweat with colorimetric wearable biosensors $\uparrow$
}

\begin{abstract}
Andreu Vaquer, (D) Enrique Barón (D) * and Roberto de la Rica (D) *
Low glucose levels during exercise may lead to hypoglycemia, which can have grave consequences in diabetic athletes. Mobile colorimetric wearable biosensors that measure glucose levels in sweat are ideal for self-monitoring as they can utilize the camera in smartphones for signal reading. However, colorimetric biosensors proposed thus far have higher limit of detection (LOD) than electrochemical devices, which makes them unsuitable for detecting hypoglycemia. In this manuscript we describe colorimetric wearable biosensors that detect glucose in sweat with an LOD of $0.01 \mathrm{mM}$ and a dynamic range up to $0.15 \mathrm{mM}$. The devices are made of filter paper and incorporate a sweat volume sensor and a color chart for signal correction. The biosensors do not suffer from interferences originated by delayed sample readings, or differences in bending angle and sample $\mathrm{pH}$. When applied to volunteers performing an exercise routine, sweat glucose levels corrected with sweat volume measurements correlated well with blood glucose measurements performed with a commercial device. The devices are lightweight and easily disposable. These features, along with the smartphone-based colorimetric readout, makes them promising as "over-the-counter" tests for measuring glucose levels non-invasively during exercise.
\end{abstract}

Received 15th February 2021, Accepted 18th March 2021

DOI: 10.1039/d1an00283j rsc.li/analyst biomarker in sweat with a lower limit of detection. Such devices could combine the benefits of colorimetric readouts with the high sensitivity that is currently only attainable with electrochemical devices. ${ }^{2,12,14,16}$

In this manuscript we describe a wearable analytical platform with colorimetric readout that detects glucose in sweat with a limit of detection of $0.01 \mathrm{mM}$. It consists of a biosensor for measuring glucose, a sensor for determining the sweat volume, and a color chart for signal normalization (Fig. 1). The sensors are made of filter paper and are worn by fixing them on the skin with adhesive medical tape, where they absorb sweat by capillarity through the inlet (Fig. 1A). In the glucose biosensor, sweat carries the enzymes glucose oxidase (GOX) and horseradish peroxidase (HRP) to the detection zone (Fig. 1B). There, the chromogen TMB is oxidized by HRP using the $\mathrm{H}_{2} \mathrm{O}_{2}$ produced by GOX during the oxidation of glucose. This design, which relies in mobilizing enzymes to a colorimetric transducer, reduces background signals that may compromise the detection of low concentrations of glucose. ${ }^{17}$ The biosensor shape was also fine-tuned to improve the LOD and precision. The sweat volume sensor is a paper strip modified with pegylated gold nanoparticles (AuNPs) that allows estimating the volume of sample entering the platform (Fig. 1A). The nanoparticles are stored in a reservoir containing polystyrene sulfonate (PSS), which prevents irreversible interactions with the paper even in dry conditions. ${ }^{18}$ Thanks to this, the nanoparticles are carried through the paper as the wearer sweats,
Multidisciplinary Sepsis Group, Health Research Institute of the Balearic Islands (IdISBa), Son Espases University Hospital, o7120 Palma de Mallorca, Spain.

E-mail: Enrique.baron@ssib.es, Roberto.delarica@ssib.es

$\dagger$ Electronic supplementary information (ESI) available. See DOI: 10.1039/ d1an00283j 


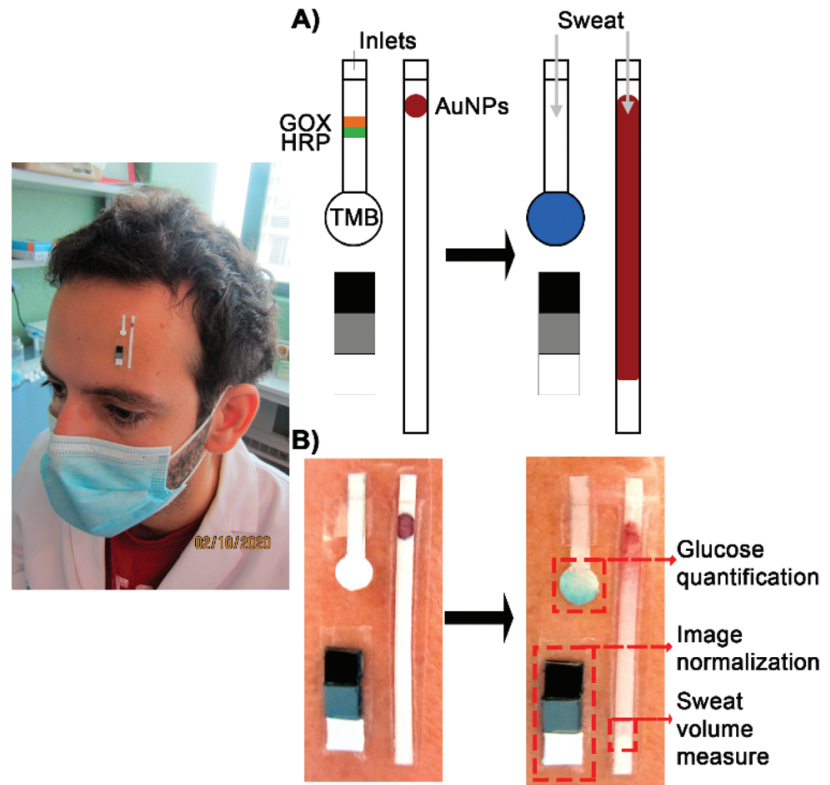

Fig. 1 Design of the wearable device. (A) Schematic representation. The paper strips are isolated from the skin thanks to a medical adhesive underneath it. Sweat enters the sensors through the inlet, which is the only part in contact with the skin. The color reference chart allows to account for differences in light conditions between different measures. (B) Photographs of a real application of the device before and after sweating.

thus creating a red-colored trail that is related to the volume of sweat entering the biosensor (Fig. 1A and B). The color chart in the platform is used as a reference for normalizing the color of pictures taken with a smartphone, which enables signal quantification in different ambient light conditions. The device yields consistent results at different sweat volumes, bent angles or sweat pH values. When applied to 9 healthy volunteers during an exercise routine, sweat glucose levels correlated well with blood glucose levels measured with a commercial glucometer. This paves the way for using our wearable analytical platform as an alternative to invasive methods for glucose monitoring during exercise.

\section{Experimental}

\section{Materials}

D-(+)-Glucose ( $\geq 99.5 \%), 3,3^{\prime}, 5,5^{\prime}$-tetramethylbenzidine (TMB, $\geq 99 \%$ ), horseradish peroxidase (HRP, Type VI-A, salt free), glucose oxidase (GOX) from Aspergillus niger (100 000-250 000 $\mathrm{U} \mathrm{g}^{-1}$, Type X-S), Tween 20, Whatman filter paper Grade 6 ( $3 \mu \mathrm{m}$ pore, cellulose, ashless), Grade 1 (11 $\mu \mathrm{m}$ pore, cellulose, ashless), Grade 41 (20 $\mu \mathrm{m}$ pore, cellulose, ashless), poly (sodium 4-styrenesulfonate) (PSS, 30\% in water), poly(ethylene glycol) 2-mercaptoethyl ether acetic acid (PEG-COOH, $M_{\mathrm{n}}$ $2100)$, sodium citrate tribasic dehydrate ( $\geq 99.0 \%)$ and gold(III) chloride hydrate (99.995\%) were purchased from SigmaAldrich. Bovine Serum Albumin (BSA, protease free) was pur- chased from VWR. Phosphate buffered saline (PBS) containing $1 \mathrm{mg} \mathrm{mL}{ }^{-1}$ BSA and $0.05 \%$ Tween was prepared following standard procedures. Polyethylene glycol coated gold nanoparticles (PEG-AuNPs, $20 \mathrm{~nm}$ ) were fabricated as described elsewhere. ${ }^{19}$

\section{Fabrication of glucose biosensor}

For the rectangular design, Whatman filter paper (Grade 41, $20 \mu \mathrm{m}$ ) was manually cut into $1.5 \mathrm{~cm} \times 0.2 \mathrm{~cm}$ strips and modified with a solution of BSA-Tween $\left(1 \mathrm{mg} \mathrm{mL}^{-1}\right.$ and $0.05 \%$ in $\mathrm{PBS}$, respectively) to avoid non-specific interactions between enzymes and the paper substrate. TMB (10 mM) was drop casted $(0.5 \mu \mathrm{L})$ at the end of the strip. As for the circular design a circular area with a $0.5 \mathrm{~cm}$ diameter was included. Strips had a total length of $1.5 \mathrm{~cm}$. In this case TMB was drop casted in the circular area, which admitted more volume $(1 \mu \mathrm{L})$. In both cases HRP $\left(50 \mu \mathrm{g} \mathrm{mL}^{-1}\right.$ in PBS) and GOX (10 $\mathrm{mg} \mathrm{mL}^{-1}$ in PBS) were drop casted $3 \mathrm{~mm}$ away from the TMB area (Fig. 1A). GOX concentration was optimized to obtain the highest colorimetric signal (Fig. S1†).

The entry point for sweat is a $0.3 \mathrm{~cm} \times 0.2 \mathrm{~cm}$ inlet in contact with the skin. Sweat flows through the device by capillary action. Due to the sample flow, enzymes arrive to the detection area at the same time as glucose. The rest of the device is covered with medical adhesive to isolate it from the skin (3 M, Minnesota, USA).

\section{Fabrication of sweat volume sensors}

Grade 41 filter paper $(20 \mu \mathrm{m})$ from Whatman was manually cut into $3.5 \mathrm{~cm} \times 0.2 \mathrm{~cm}$ strips and modified with BSA-Tween ( $1 \mathrm{mg} \mathrm{mL} \mathrm{m}^{-1}$ and $0.05 \%$ in PBS, respectively) to avoid nonspecific interactions. Afterwards, $0.5 \mu \mathrm{L}$ of PSS (10\%) was added $2 \mathrm{~mm}$ away from the inlet and dried. Finally, AuNPs (0.5 $\mu \mathrm{L}$ of a $1: 2$ dilution) were added in the same spot. An inlet of $0.3 \mathrm{~cm} \times 0.2 \mathrm{~cm}$ serves as the entry point for sweat, which flows through the sensor by capillary action (Fig. 1A and B). The sensor was calibrated by measuring the distance from the inlet to the flow end line after the addition of PBS volumes between 2.5 and $8 \mu \mathrm{L}$. The rest of the device is covered with medical adhesive to isolate it from the skin (3 M, Minnesota, USA).

\section{Colorimetric detection}

Pixel intensity (PI) profiles were obtained using ImageJ. Colorimetric signal $(S)$ was calculated by subtracting the pixel intensity from 255 (pure white in grayscale). This yields an inverted signal in comparison to the raw data. When $S$ is corrected by the sample volume measured with the sweat sensor, it is expressed as $S / V$ to indicate that it has been divided by the volume. During this project images were obtained using two different methods: scanning the paper strips using an MFC-1910 W scanner from Brother or using a Huawei P30 lite smartphone. Regarding the later, changes in light conditions were compensated using a 3-color reference chart made of photographic cards (see Fig. 1A and B). Both methods are compared in Fig. S2. $\dagger$ 
Volume-corrected detection of sweat glucose with the wearable analytical platform

The device was applied to 9 healthy volunteers during a stationary bicycle routine. All experiments were performed in accordance with the Health Research Institute of the Balearic Islands (IdISBa) Guidelines and approved by the Balearic Islands Ethics Committee (IB 4053/19 PI). Informed consents were obtained from all human participants of this study.

It consisted of a 15 min warm-up followed by gradual increases of the workload. A commercial glucometer (FreeStyle Optimum Neo, Abbott) was used to monitor blood glucose at different times. After a first blood measure to assess the basal level, the workload was increased gradually every 10 minutes. After the first sweat measure, volunteers were fed a commercial energy gel containing a high dose of glucose (total dose of $c a .4 \mathrm{~g}$ ). This was done in order to confirm that our device could monitor sweat glucose changes and that these changes correlated well with blood changes, as previously observed with other methods. ${ }^{14,20-22}$

\section{Results and discussion}

\section{Glucose biosensor design}

In the glucose biosensor design shown in Fig. 1A sweat carries enzymes from a reservoir to a detection area. This separation between enzymes and chromogen was necessary because mixing them together in the same area resulted in the spontaneous generation of color, which could interfere in the posterior detection of glucose (Fig. S3 $\uparrow$ ). The biosensors were fabricated with Whatman paper with a pore size of $20 \mu \mathrm{m}$ because devices fabricated with filter paper with a small pore size ( $3 \mu \mathrm{m}$ and $11 \mu \mathrm{m}$ ) yielded lower signals (Fig. S4 $\dagger$ ). With papers of smaller pore sizes, the flow rate is slower and therefore less enzymes arrive to the detection zone, resulting in a lower sensitivity and loss of linearity. ${ }^{17}$ Our first prototype consisted in a paper strip with a continuous rectangular tip, which contained the detection zone modified with TMB (Fig. 2A). Enzymes were placed above the detection zone and
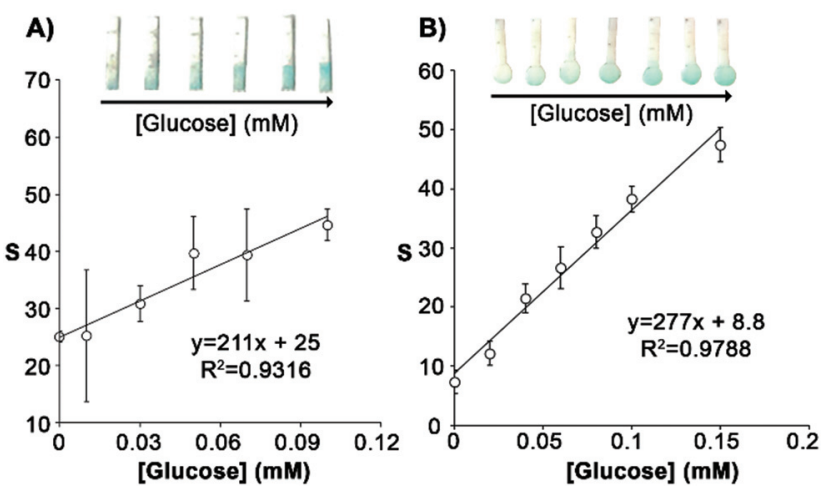

Fig. 2 Impact of biosensor shape on the colorimetric signal S. (A) Calibration of the rectangular tip design. (B) Calibration of the circular tip design. Error bars represent the SD of three biosensors. carried there by the sample flow as shown in Fig. 1. This prototype was calibrated by adding a fixed volume $(3 \mu \mathrm{L})$ of solutions containing glucose at different concentrations ( 0 to $0.1 \mathrm{mM}$ ). In Fig. 2A, the rectangular tip becomes blue as the concentration of glucose increases. The corresponding calibration shows a linear trend, with a limit of detection expressed as the signal above 3 times the standard deviation of the blank (3SD criterion) of $0.008 \mathrm{mM}$. However, the deviation obtained among the different replicates for the same sample concentration was very high and thus the biosensor was unsuitable for monitoring sweat glucose levels precisely. We hypothesized that this could be originated by an inefficient transport of enzymes to the detection zone due to the small volume added $(3 \mu \mathrm{L})$, as shown for paper substrates with smaller pore sizes (Fig. S4†). Attempts at calibrating the biosensors with higher sample volumes were unsuccessful because the biosensor area was too small to absorb them. We then hypothesized that a round tip could solve this problem as it would increase the detection area, thus allowing more sample to flow in. In turn, this should improve the transport of reagents to the detection zone and yield higher signals. In Fig. 2B, biosensors with a round tip were calibrated with solutions containing different concentration of glucose and a fixed volume of $7 \mu \mathrm{L}$. The plot shows a linear trend with a slope higher than the one obtained with the squared design (277 vs. 202, respectively), which agrees well with the idea that a larger tip increases signals by adsorbing more sample. In addition, the new prototype has still a low limit of detection $(0.01 \mathrm{mM})$ and considerably reduces the standard deviation of 3 independent measurements obtained with different devices. Furthermore, the dynamic range $(0.01-0.15 \mathrm{mM})$ fits well with the sweat glucose physiological range $(0.04-0.1 \mathrm{mM})$ and also covers the hypoglycemic threshold in type 1 diabetes $(<0.03 \mathrm{mM}),{ }^{23}$ which is promising for measuring variations of this biomarker under pathological conditions. Thanks to the sweat volume sensor, the sample volume can be monitored to avoid the saturation of the glucose biosensor. Once the nanoparticles indicate that the desired sample volume has entered the sensor, the device is removed from the skin to prevent saturation.

\section{Volume and color corrections}

The low LOD shown in Fig. 2 was obtained with a fixed sample volume and with a tabletop scanner, which fixes ambient light conditions. However, in real applications variations in these parameters could compromise the performance of glucose measurements. Our wearable analytical platform incorporates a sweat volume sensor and a reference color chart in order to compensate for variations in sample volume and changing light conditions (Fig. 1). Monitoring the sample volume entering the biosensor is crucial because as the volume of sample increases, the amount of sample and enzymes that arrive to the detection zone also increases. This is shown in Fig. 3A, where increasing the volume of a solution containing $0.1 \mathrm{mM}$ glucose increases the colorimetric signal generated by the biosensor. With the present design, the sample volume is calculated with the sensors shown in Fig. 3B. In this figure, there is 

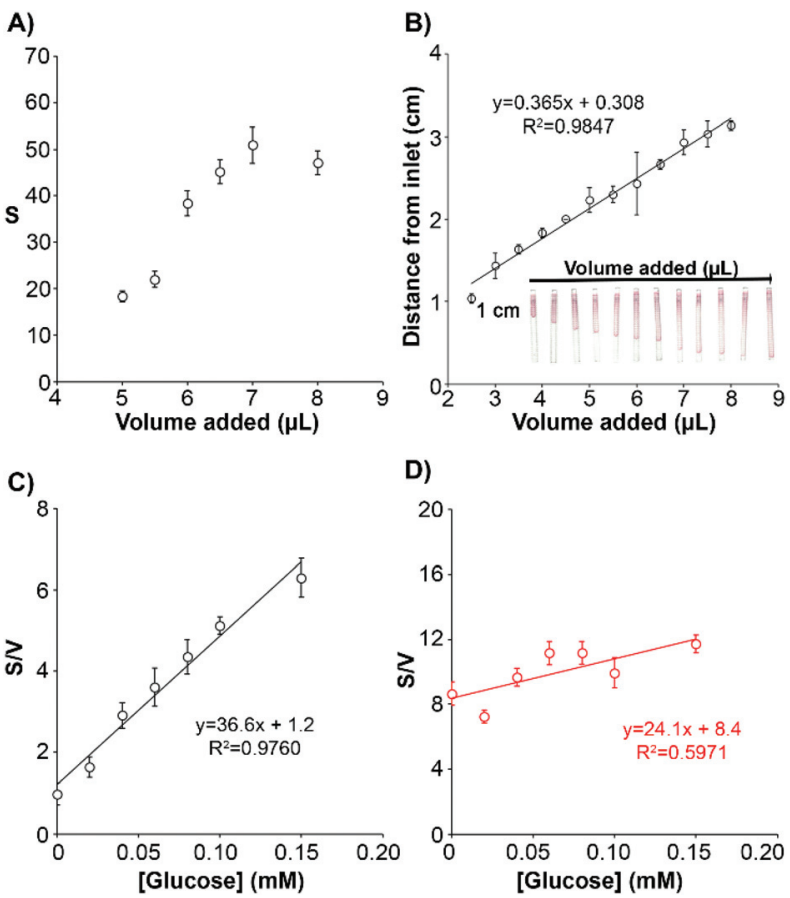

D)

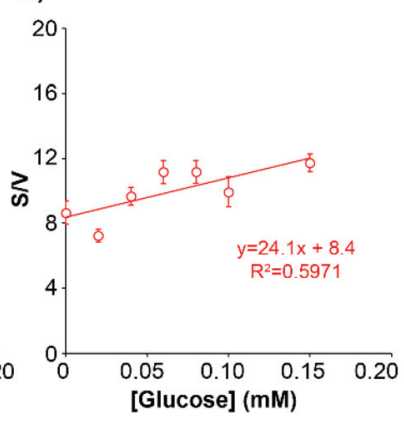

Fig. 3 Impact of volume and color correction on the detection of glucose. (A) colorimetric signal $\mathrm{S}$ obtained with the glucose biosensor after adding different volumes of the same glucose solution. (B) Calibration of the volume sensor. (C) Calibration of the glucose biosensor with different glucose concentrations corrected with the measured volume from the sweat volume sensor. (D) Same calibration as in (C) but without color normalization. Error bars represent the SD of three different devices.

a linear relationship between the distance travelled by gold nanoparticles and the added volume, which validates the proposed sensor for determining the sample volume in the range between 2.5 and $8 \mu \mathrm{L}$. This range can be fine-tuned by changing the width and length of the channel, making it a versatile approach for determining the sweat volume. ${ }^{17}$ Next, we tested the impact of incorporating sweat volume measurements in the sensitivity and limit of detection of the glucose biosensor. In Fig. 3C, when the signal was divided by the volume measured with the sweat sensor the calibration plot shows a linear trend between 0 and $0.15 \mathrm{mM}$, which is the same dynamic range reported in Fig. 2B. The limit of detection (3SD criterion) is $0.01 \mathrm{mM}$, which is the same as the prototype without sample volume correction. The dynamic range is also the same as above. These experiments demonstrate that correcting the glucose signal using sweat volume measures does not compromise the analytical performance of the wearable platform. The colorimetric signal in Fig. 3C was calculated by taking photographs with a smartphone under varying illumination conditions and normalizing the colorimetric signals with the 3-color reference chart. In Fig. 3D raw data without color normalization yielded a poor linear fit with a smaller slope, thus demonstrating that color normalization is crucial for signal quantification.

\section{Analysis of potential interferences}

After overcoming issues related to varying sample volume and light conditions we sought to test the influence of other potential interferences, including the time elapsed from signal generation to signal reading, changes in sweat $\mathrm{pH}$, and differences in biosensor bending angles. For example, in enzymatic signal generation schemes the signal increases with time until saturation is reached. ${ }^{24}$ This may introduce unwanted variability in the measurements when reading the colorimetric signal if time is not finely controlled. Fig. $4 \mathrm{~A}$ and Fig. $\mathrm{S} 5 \dagger$ show that the colorimetric signal remains stable through time after 3 minutes for all the concentrations of glucose assayed. These experiments demonstrate that with our biosensors it is not necessary to exert a fine control over the signal acquisition time in order to obtain reproducible results as long as at least 3 minutes have passed since the sample entered the biosensor.

With regards to the bending angle, it is well known that during the application of the device on the skin the wearable biosensor may bend and that this could limit its performance. ${ }^{25}$ Bending could affect the transport of fluids by capillarity. In turn, this could vary the amount of analyte and reagents being dragged to the detection area, which would make the measurements less reproducible. In this work we applied the device to the forehead, where a maximum bending of $40^{\circ}$ was estimated could happen. In Fig. $4 \mathrm{~B}$ our device performs well at all angles when tested at $0^{\circ}, 20^{\circ}$ and $40^{\circ}$. A $16 \%$ decrease in the slope of the calibration curve was detected when the angle increased to $40^{\circ}$. Although this variation was not statistically significant $(p>0.05)$, it suggested that extreme angles may have some impact on the detection of sweat biomarkers. Changes in sweat $\mathrm{pH}$ are another potential source of variability. The enzymatic activity of both HRP and GOX can be affected by the $\mathrm{pH}$ of sweat, which has been reported to change between 4.5 and $7 .^{26}$ To study this, we prepared 3 calibration curves with different glucose dilutions in an artificial sweat matrix adjusted to $\mathrm{pH} \mathrm{5,6}$ and 7 (Fig. 4C). The calibration plots were nearly identical, showing the same slope at the three $\mathrm{pH}$ values assayed (CV 4.2\%). These results demonstrate that changes in $\mathrm{pH}$ do not contribute to the variability in the measurements.

\section{Volume-corrected detection of sweat glucose with the wearable analytical platform}

Next we tested the wearable analytical platform implementing the sweat volume and glucose sensors for measuring variations in glucose levels during an exercise routine. The sweat volume correction considers that the inlets of both sensors are close enough to collect an equivalent sample volume, as previously shown in other wearable devices featuring more than one inlet for volume correction. ${ }^{10,27,28}$ The routine began with a $15 \mathrm{~min}$ warm-up followed by an increase of the workload every 10 minutes. Basal sweat glucose was determined prior to the intake of an energy gel. The gel was provided to boost blood glucose levels which would translate into an increase of sweat 


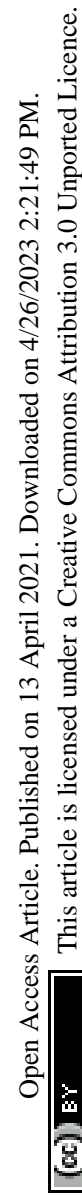

A)

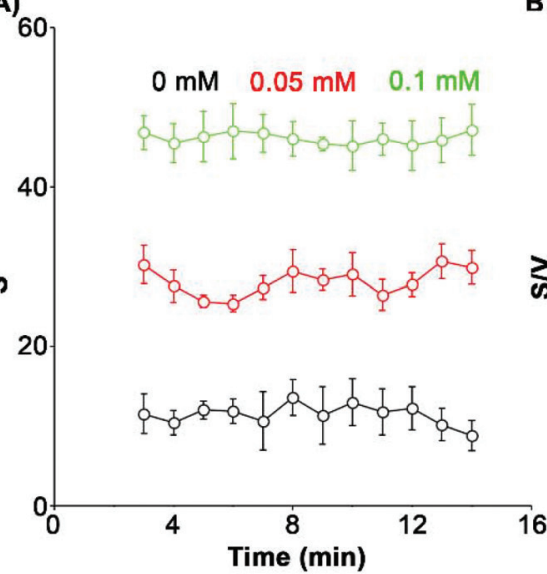

B)

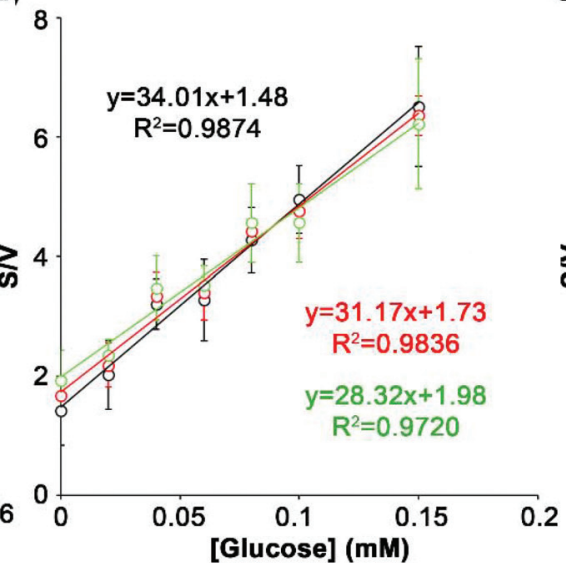

C)

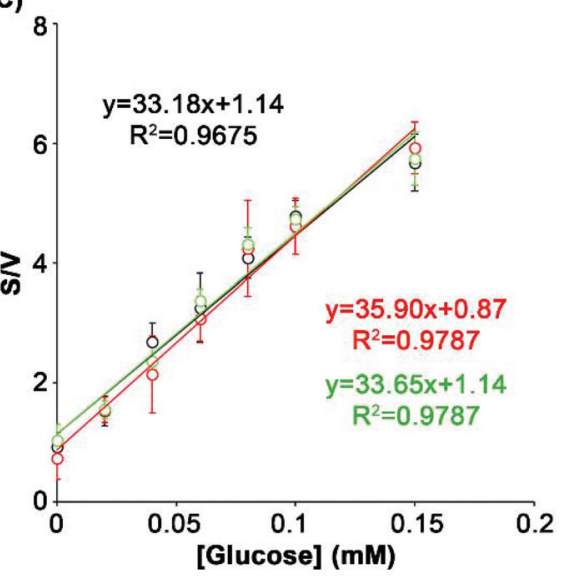

Fig. 4 Impact of variations in signal acquisition time, bending angles and sweat $\mathrm{pH}$ on the colorimetric signal. (A) Variation of $\mathrm{S}$ when the signal acquisition time is delayed for 3 different glucose concentrations. (B) Calibration curves at three different bend angles: $0^{\circ}$ (black), $20^{\circ}$ (green) and $40^{\circ}$ (red). (C) Calibration curves at three different pH values: 5 (red), 6 (green) and 7 (black). Error bars represent the SD of three sensors in all cases.

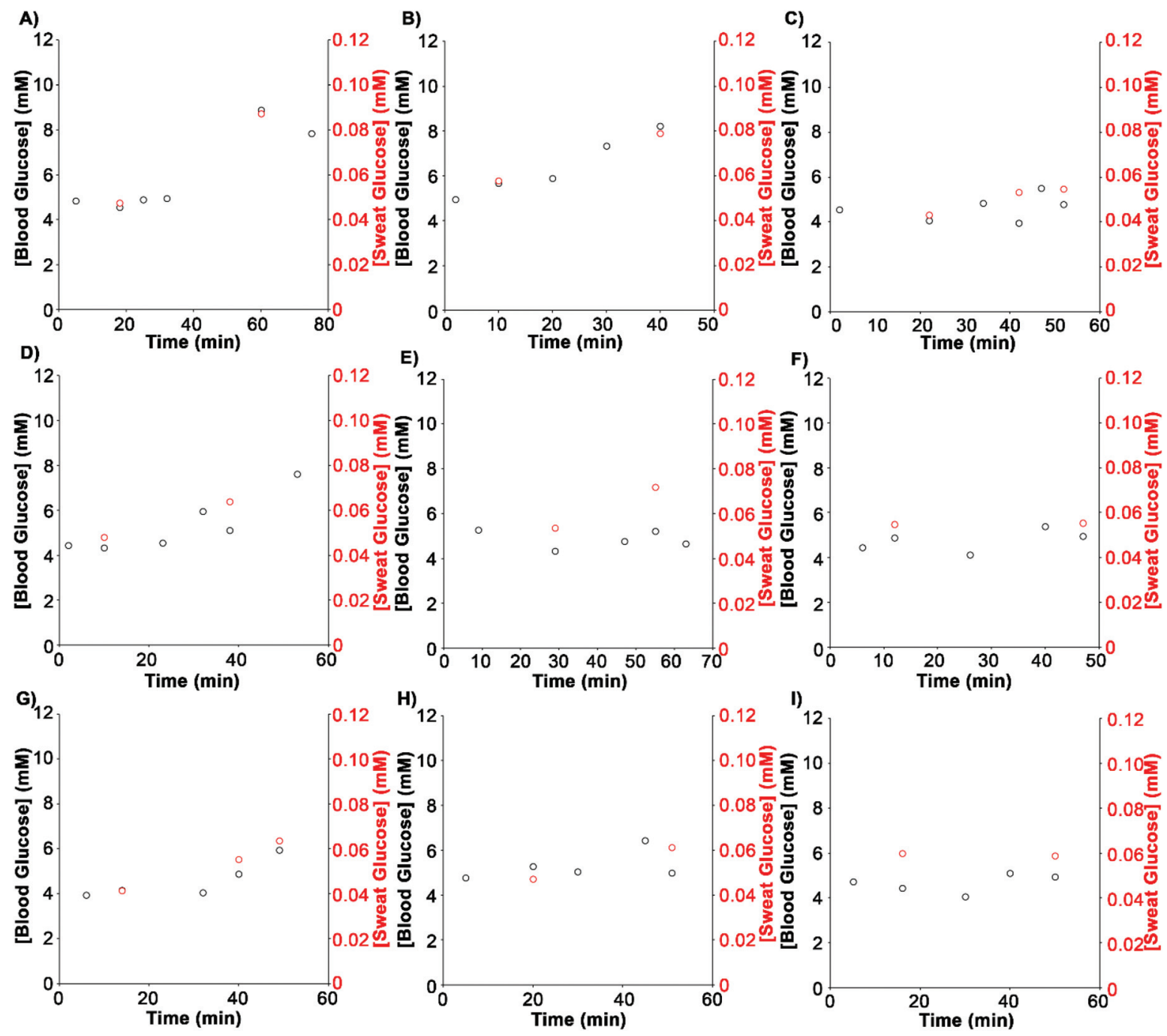

Fig. 5 Detection of sweat glucose levels with the analytical platform shown in Fig. 1 (red) or blood glucose with a commercial glucometer (black) in samples obtained from 9 different healthy volunteers $(A-I)$ 


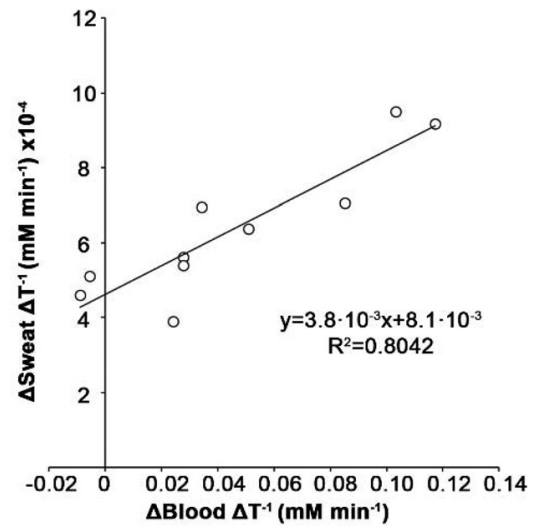

Fig. 6 Correlation between glucose concentration in blood (glucometer) and in sweat (our device).

glucose levels. To obtain the concentration of glucose in sweat, colorimetric signals from the glucose biosensor were corrected with the color chart and divided by the sweat volume. Then they were interpolated in a calibration plot performed at 37 degrees to simulate the body temperature (Fig. S6 $\dagger$ ). Fig. 5 shows variations in glucose levels in blood with time measured with a commercial glucometer, and the same variations in sweat measured with our wearable device. Both methods yielded comparable measurements, with glucose concentrations approximately 100 times lower in sweat than in blood as expected from previous reports in the literature. ${ }^{14,15}$ These experiments show that blood glucose analyses during an exercise routine can be substituted by non-invasive determinations in sweat performed with the proposed wearable analytical platform.

Remarkably, the same sweat glucose measurements without sweat volume correction showed no correlation with blood levels, which validates our multisensor design for quantifying glucose levels during exercise (Fig. S7†). This also shows that, although the sweat volume and glucose sensors may not capture the exact same amount of sweat, differences are small enough to enable accurate measurements. Fig. 6 shows a statistically significant correlation between sweat glucose levels measured with our device and blood glucose levels measured with the commercial test. The correlation coefficient $\left(R^{2}=\right.$ 0.8042 ) agrees with other studies performed with electrochemical sensors, which range from 0.75 to $0.85 .^{14,29-31}$

\section{Conclusions}

In conclusion, a colorimetric wearable device for the detection of low sweat glucose concentrations has been developed. It incorporates a glucose biosensor with minimal interference from signal reading time, bending angle or sweat $\mathrm{pH}$ with a companion sensor for estimating the sample volume and a reference chart to eliminate the influence of varying light conditions. Measuring the sweat volume allows to correct variations in the signal originating from different sweat rates. The combined platform has a low LOD of $0.01 \mathrm{mM}$, making it suitable for detecting hypoglycemia. Sweat glucose levels correlated well with blood glucose levels, which is promising for using our approach as a non-invasive alternative for glucose monitoring during exercise. Compared to other colorimetric devices made of different ploymers ${ }^{28}$ the proposed biosensors are entirely made of paper. This makes them easy to dispose of, making them ideal as single-use devices. They are also lightweight and can be easily fixed onto the skin with medical grade tape. These features, along with the smartphone based colorimetric signal transduction mechanism, pave the way for its widespread use as an "over-the-counter" biosensing device with a companion app for color quantification.

\section{Author contributions}

CRediT author statement: Andreu Vaquer: Methodology, validation, investigation. Enrique Barón: Methodology, validation, writing-original draft, writing - review \& editing; Roberto de la Rica: Conceptualization, methodology, resources, writing-original draft, writing - review \& editing, supervision, project administration, funding acquisition.

\section{Conflicts of interest}

R. R. discloses that a patent application describing the method for storing nanoparticles in paper reservoirs has been filed (PCT/EP2020/075013).

\section{Acknowledgements}

R. R. and E. B. acknowledge funding from IdISBa/Impost turisme sostenible/Agència d'Estratègia Turística de les Illes Balears-Govern de les Illes Balears through a Radix and Folium fellow-ships, respectively. E. B. acknowledges funding from the Instituto de Salud Carlos III (Sara Borrell contract). A. V. acknowledges funding from European Social Fund (ESF) through JoTReSdOS program. Open access is granted by funds from the Liberi program (IdISBa).

\section{References}

1 K. Guk, G. Han, J. Lim, K. Jeong, T. Kang, E. K. Lim and J. Jung, Nanomaterials, 2019, 9, 813.

2 J. Kim, A. S. Campbell, B. E. F. de Ávila and J. Wang, Nat. Biotechnol., 2019, 37, 389-406.

3 M. Chung, G. Fortunato and N. Radacsi, J. R. Soc., Interface, 2019, 16(159), 20190217.

4 M. G. Wolfe, M. M. Ali and J. D. Brennan, Anal. Chem., 2019, 91, 4735-4740.

5 X. Luo, J. Xia, X. Jiang, M. Yang and S. Liu, Anal. Chem., 2019, 91, 15461-15468. 
6 E. F. M. Gabriel, P. T. Garcia, T. M. G. Cardoso, F. M. Lopes, F. T. Martins and W. K. T. Coltro, Analyst, 2016, 141, 47494756.

7 V. P. Rachim and W. Y. Chung, Sens. Actuators, B, 2019, 286, 173-180.

8 H. Jang, J. H. Park, J. Oh, K. Kim and M. G. Kim, ACS Sens., 2019, 4, 1103-1108.

9 J. Choi, R. Ghaffari, L. B. Baker and J. A. Rogers, Sci. Adv., 2018, 4, 1-10.

10 A. Koh, D. Kang, Y. Xue, S. Lee, R. M. Pielak, J. Kim, T. Hwang, S. Min, A. Banks, P. Bastien, M. C. Manco, L. Wang, K. R. Ammann, K. I. Jang, P. Won, S. Han, R. Ghaffari, U. Paik, M. J. Slepian, G. Balooch, Y. Huang and J. A. Rogers, Sci. Transl. Med., 2016, 8, 1-14.

11 J. Xiao, Y. Liu, L. Su, D. Zhao, L. Zhao and X. Zhang, Anal. Chem., 2019, 91, 14803-14807.

12 M. Bariya, H. Y. Y. Nyein and A. Javey, Nat. Electron., 2018, 1, 160-171.

13 S. R. Heller, Diabetes Care, 2017, 40, 155-157.

14 Y. J. Hong, H. Lee, J. Kim, M. Lee, H. J. Choi, T. Hyeon and D. H. Kim, Adv. Funct. Mater., 2018, 28, 1-12.

15 H. Lee, T. K. Choi, Y. B. Lee, H. R. Cho, R. Ghaffari, L. Wang, H. J. Choi, T. D. Chung, N. Lu, T. Hyeon, S. H. Choi and D. H. Kim, Nat. Nanotechnol., 2016, 11, 566572.

16 X. Zhang, Y. Jing, Q. Zhai, Y. Yu, H. Xing, J. Li and E. Wang, Anal. Chem., 2018, 90, 11780-11784.

17 A. Vaquer, E. Baron and R. de la Rica, ACS Sens., 2021, 6, 130-136.

18 A. Alba-Patiño, C. Adrover-Jaume and R. D. La Rica, ACS Sens., 2020, 5, 147-153.

19 R. De La Rica, Nanoscale, 2017, 9, 18855-18860.
20 E. Cho, M. Mohammadifar and S. Choi, Micromachines, 2017, 8(9), 265-276.

21 H. Y. Y. Nyein, M. Bariya, L. Kivimäki, S. Uusitalo, T. S. Liaw, E. Jansson, C. H. Ahn, J. A. Hangasky, J. Zhao, Y. Lin, T. Happonen, M. Chao, C. Liedert, Y. Zhao, L. C. Tai, J. Hiltunen and A. Javey, Sci. Adv., 2019, 5(8), eaaw9906.

22 J. Kim, A. S. Campbell and J. Wang, Talanta, 2018, 177, 163-170.

23 D. J. Cox, B. Kovatchev, D. Koev, L. Koeva, S. Dachev, D. Tcharaktchiev, A. Protopopova, L. Gonder-Frederick and W. Clarke, Int. J. Behav. Med., 2004, 11, 212-218.

24 Y. Yang, E. Noviana, M. P. Nguyen, B. J. Geiss, D. S. Dandy and C. S. Henry, Anal. Chem., 2017, 89, 71-91.

25 G. Li and D. Wen, J. Mater. Chem. B, 2020, 8, 3423-3436.

26 A. Wiorek, M. Parrilla, M. Cuartero and G. A. Crespo, Anal. Chem., 2020, 92, 10153-10161.

27 J. Choi, Y. Xue, W. Xia, T. R. Ray, J. T. Reeder, A. J. Bandodkar, D. Kang, S. Xu, Y. Huang and J. A. Rogers, Lab Chip, 2017, 17, 2572-2580.

28 J. Choi, A. J. Bandodkar, J. T. Reeder, T. R. Ray, A. Turnquist, S. B. Kim, N. Nyberg, A. Hourlier-Fargette, J. B. Model, A. J. Aranyosi, S. Xu, R. Ghaffari and J. A. Rogers, ACS Sens., 2019, 4, 379-388.

29 J. Moyer, D. Wilson, I. Finkelshtein, B. Wong and R. Potts, Diabetes Technol. Ther., 2012, 14, 398-402.

30 S. K. Garg, R. O. Potts, N. R. Ackerman, S. J. Fermi, J. A. Tamada and H. P. Chase, Diabetes Care, 1999, 22, 1708-1714.

31 E. V. Karpova, E. V. Shcherbacheva, A. A. Galushin, D. V. Vokhmyanina, E. E. Karyakina and A. A. Karyakin, Anal. Chem., 2019, 91, 3778-3783. 